\title{
Baker's Explicit abc-Conjecture and Waring's problem
}

\author{
Shanta Laishram
}

\begin{abstract}
The conjecture of Masser-Oesterlé, popularly known as abc-conjecture has many consequences. We show that Waring's problem is a consequence of an explicit version of $a b c$-conjecture due to Baker.

Keywords. ABC Conjecture, Waring's problem.

2010 Mathematics Subject Classification. 11D41, 11D75, 11E76, 11P05
\end{abstract}

\section{Introduction}

For any positive integer $i>1$, let $N=N(i)=\prod_{p \mid i} p$ be the radical of $i, P(i)$ be the greatest prime factor of $i$ and $\omega(i)$ be the number of distinct prime factors of $i$ and we put $N(1)=1, P(1)=1$ and $\omega(1)=0$. The well known conjecture of Masser-Oesterlé states that

Conjecture 1.1. abc-conjecture of Masser and Oesterlé: For any given $\epsilon>0$ there exists a computable constant $\mathfrak{c}_{\epsilon}$ depending only on $\epsilon$ such that if

$$
a+b=c
$$

where $a, b$ and $c$ are coprime positive integers, then

$$
c \leq \mathfrak{c}_{\epsilon}\left(\prod_{p \mid a b c} p\right)^{1+\epsilon} .
$$

This is popularly known as $a b c-$ conjecture. The $a b c-$ conjecture has already become well known for the number of interesting consequences it entails. Many famous conjectures and theorems in number theory would follow immediately from the $a b c$-conjecture. An explicit version of this conjecture due to Baker [Bak94] is the following:

Conjecture 1.2. Explicit $a b c$-conjecture: Let $a, b$ and $c$ be pairwise coprime positive integers satisfying (1.1). Then

$$
c<\frac{6}{5} N \frac{(\log N)^{\omega}}{\omega !}
$$

where $N=N(a b c)$ and $\omega=\omega(N)$.

We observe that $N=N(a b c) \geq 2$ whenever $a, b, c$ satisfy (1.1). We shall refer to Conjecture 1 . as $a b c-$ conjecture and Conjecture 1 . as explicit $a b c-$ conjecture. We have 
Theorem 1. (Laishram and Shorey [LaSh12] ) Assume Conjecture 1. Let $a, b$ and $c$ be pairwise coprime positive integers satisfying (1.1) and $N=N(a b c)$. Then we have

$$
c<N^{1+\frac{3}{4}} .
$$

Further for $0<\epsilon \leq \frac{3}{4}$, there exists $\omega_{\epsilon}$ depending only $\epsilon$ such that when $N=N(a b c) \geq N_{\epsilon}=\prod_{p \leq p_{\omega_{\epsilon}}} p$, we have

$$
c<\kappa_{\epsilon} N^{1+\epsilon}
$$

where

$$
\kappa_{\epsilon}=\frac{6}{5 \sqrt{2 \pi \max \left(\omega, \omega_{\epsilon}\right)}} \leq \frac{6}{5 \sqrt{2 \pi \omega_{\epsilon}}}
$$

with $\omega=\omega(N)$. Here are some values of $\epsilon, \omega_{\epsilon}$ and $N_{\epsilon}$.

\begin{tabular}{|c|c|c|c||c|c|c|c|}
\hline$\epsilon$ & $\frac{3}{4}$ & $\frac{7}{12}$ & $\frac{6}{11}$ & $\frac{1}{2}$ & $\frac{34}{71}$ & $\frac{5}{12}$ & $\frac{1}{3}$ \\
\hline$\omega_{\epsilon}$ & 14 & 49 & 72 & 127 & 175 & 548 & 6460 \\
\hline$N_{\epsilon}$ & $e^{37.1101}$ & $e^{204.75}$ & $e^{335.71}$ & $e^{679.585}$ & $e^{1004.763}$ & $e^{3894.57}$ & $e^{63727}$ \\
\hline
\end{tabular}

Thus $c<N^{2}$ which was conjectured in Granville and Tucker [GrTu02].

\section{Ideal Waring's Conjecture}

For each integer $k \geq 2$, denote by $g(k)$ the smallest integer $g$ such that any positive integer is the sum of at most $g$ integers of the form $x^{k}$. A result of Euler implies that a lower bound for $g(k)$ is $2^{k}+\left\lfloor(3 / 2)^{k}\right\rfloor-2$. The so-called Ideal Waring's Conjecture is the following conjecture, dating back to 1853 :

Conjecture 2.1. For any $k \geq 2$, the equality $g(k)=2^{k}+\left\lfloor\left(\frac{3}{2}\right)^{k}\right\rfloor-2$ holds.

Theorem 2. Assume Conjecture 1.. Then Conjecture 2. is true.

Conjecture 2. has a long and interesting history. We refer to Waldschmidt [Mic00, pp 12] for further details. We prove Theorem 2. in the next section.

\section{Proof of Theorem 2 .}

We write

$$
3^{k}=2^{k} q+r \text { with } 0<r<2^{k} \text { and } q=\left\lfloor\left(\frac{3}{2}\right)^{k}\right\rfloor .
$$

L. E. Dickson and S.S. Pillai (see for instance [HaWr54, Chap. XXI] or [Nar86, p. 226 Chap. IV]) proved independently in 1939 that the ideal Waring's Conjecture(Conjecture 2.) holds provided that the remainder $r=3^{k}-2^{k} q$ satisfies

$$
r \leq 2^{k}-q-3 .
$$

The condition (3.3) is satisfied for $3 \leq k \leq 471600000$ as well as for sufficiently large $k$, as shown by K. Mahler [Mah57] in 1957 by means of Ridout's extension of the Thue-Siegel-Roth theorem. 
Therefore we may now suppose that $k>471600000$ and further (3.3) does not hold, i.e.,

$$
r \geq 2^{k}-q-2
$$

Let $\operatorname{gcd}\left(3^{k}, 2^{k}(q+1)\right)=3^{v}$ and set

$$
a=3^{k-v}, c=3^{-v} 2^{k}(q+1) \text { and } b=c-a=3^{-v}\left(2^{k}-r\right) .
$$

Then $a, b, c$ are relatively prime positive integers satisfying $a+b=c$ and

$$
b=3^{-v}\left(2^{k}-r\right) \leq 3^{-v}(q+3)
$$

by (3.4). Then

$$
N=N(a b c)=N\left(3^{k-v} \cdot \frac{2^{k}(q+1)}{3^{v}} \cdot b\right) \leq \frac{6 b(q+1)}{3^{v}} \leq \frac{6(q+1)(q+3)}{3^{2 v}} .
$$

First assume that $N<e^{63727}$. Then by (1.2), we have

$$
2^{k} \leq \frac{2^{k}(q+1)}{3^{v}}<N^{\frac{7}{4}}<e^{63727 \cdot \frac{7}{4}}
$$

implying

$$
k<\frac{63727 \cdot 7}{4 \cdot \log 2}<160893
$$

This is a contradiction since $k>471600000$. Therefore we may suppose that $N \geq e^{63727}$. By Theorem 1. with $\epsilon=\frac{1}{3}$ and (3.5), we have

$$
\frac{2^{k}(q+1)}{3^{v}}<\frac{6}{5 \sqrt{2 \pi \cdot 6460}}\left(\frac{6(q+1)(q+3)}{3^{2 v}}\right)^{\frac{4}{3}} .
$$

implying

$$
2^{k}<\frac{6^{\frac{7}{3}}}{5 \sqrt{12920 \pi}} q^{\frac{5}{3}}\left(1+\frac{3}{q}\right)^{\frac{5}{3}} .
$$

Since $3^{k}>2^{k} q$, we have $q<\left(\frac{3}{2}\right)^{k}$. Also $1+\frac{3}{q}<2$ since $k \geq 3$. Therefore

$$
2^{k}<\frac{6^{\frac{7}{3}} \cdot 2^{\frac{5}{3}}}{5 \sqrt{12920 \pi}}\left(\frac{3}{2}\right)^{\frac{5 k}{3}}<\left(\left(\frac{3}{2}\right)^{\frac{5}{3}}\right)^{k}<2^{k} .
$$

This is a contradiction. Hence the assertion.

\section{Acknowledgments}

The author would like to thank Michel Waldschmidt for pointing out his paper [Mic00, p. 12] to the author which led to this paper. We would like to also thank the referee for careful reading and suggestions and remarks on an earlier draft of this paper. 


\section{References}

[Bak94] A. Baker, Experiments on the abc-conjecture, Publ. Math. Debrecen 65(2004), 253-260.

[ABC3] ABC triples, page maintained by Bart de Smit at http: $/ /$ www.math.leidenuniv.nl $/ \sim$ desmit $/$ abc $/$ index.php?sort $=$ 1, see also http://rekenmeemetabc.nl/Synthese_resultaten, http ://www.math.unicaen.fr/ nitaj/abc.html.

[GrTu02] A. Granville and T. J. Tucker, It's as easy as abc, Notices of the AMS, 49(2002), 1224-31.

[HaWr54] G. H. Hardy and W. M. Wright, An introduction to the theory of numbers, Oxford Univ. Press, third ed., 1954.

[LaSh12] S. Laishram and T. N. Shorey, Baker's Explicit abc-Conjecture and applications, Acta Arith., 155 (2012), $419-429$.

[Mah57] K. Mahler, On the fractional parts of the powers of a rational number. II, Mathematika, 4(1957), 122-124.

[Nar86] W. Narkiewicz, Classical problems in number theory, Vol. 62 of Monografie Matematyczne [Mathematical Monographs], Państwowe Wydawnictwo Naukowe (PWN), Warsaw, 1986.

[Mic00] M. Waldschmidt, Perfect Powers: Pillai's works and their developments, Collected works of S. Sivasankaranarayana Plliai, Eds. R. Balasubramanian and R. Thangadurai, Collected Works Series, no. 1, Ramanujan Mathematical Society, Mysore, 2010, pp. xxii-xlvii.

Shanta Laishram

Stat Math Unit

Indian Statistical Institute

7 SJS Sansanwal Marg

New Delhi 110016, India.

e-mail: shanta@isid.ac.in 